\title{
Effects of a Novel Psychomotor Stabilizer, IRL790, on Biochemical Measures of Synaptic Markers and Neurotransmission $\$$
}

\author{
Kristina Becanovic, Maria Vittoria de Donno, Vasco C. Sousa, Joakim Tedroff, \\ and Per Svenningsson
}

Department of Clinical Neuroscience, Karolinska Institutet, Stockholm, Sweden (K.B., M.V.d.D., V.C.S., J.T., P.S.) and Integrative Research Laboratories Sweden AB, Gothenburg, Sweden (J.T.)

Received January 2, 2020; accepted April 29, 2020

\begin{abstract}
The novel small-molecule psychomotor stabilizer, IRL790, is currently in clinical trial for treatment of levodopa-induced dyskinesia and psychosis in patients with Parkinson disease. Here, we used naîve mice to investigate the effects of acute systemic administration of IRL790 on protein levels and phosphorylation states of proteins relevant for synaptic plasticity and transmission. IRL790 increased pro-brain-derived neurotrophic factor protein levels and phosphorylation at Ser1303 of the $\mathrm{N}$-methyl-D-aspartate (NMDA) subtype 2B glutamate receptor (NR2B) in prefrontal cortex. IRL790 also increased the phosphorylation states at Ser19, Ser31, and Ser40, respectively, of tyrosine hydroxylase in striatum. IRL790 reduced protein levels of the NR2B receptor in striatum but not in prefrontal cortex.
\end{abstract}

\section{Introduction}

Psychomotor stabilizers are novel treatments for neuropsychiatric disorders and neurodegenerative diseases, which form a distinct pharmacological class showing antidyskinetic, antipsychotic, and tentatively procognitive properties (Pettersson et al., 2010). They are recognized to promote their effects by modulating dopamine- and glutamate-dependent behaviors. IRL790 is primarily a dopamine $\mathrm{D}_{3}$ receptor antagonist with psychomotor-stabilizing properties in vivo (Waters et al., 2017, 2020). At higher concentrations, IRL790 also antagonizes dopamine $\mathrm{D}_{2}$ receptors. However, IRL790 has no effect on spontaneous locomotor activity over a wide dose range in the intact rat (Waters et al., 2020). In the unilateral 6- hydroxydopamine (6-OHDA) lesioned rat model, IRL790 dose-dependently reduces dyskinesias associated with prolonged levodopa treatment without affecting general locomotor activity (Waters et al., 2020). In addition to

This work was supported by the Stockholm Brain Institute (SBI), Sweden. J.T. is employed by Integrative Research Laboratories, Sweden, and is a cofounder and stockholder of the company. The other authors declare that there is no conflict of interest regarding the publication of this paper.

https://doi.org/10.1124/jpet.119.264754.

S This article has supplemental material available at jpet.aspetjournals.org.
Taken together, we report that systemically administered IRL790 rapidly elicits changes in protein level and phosphorylation state of proteins associated with a beneficial effect on synaptic markers and neurotransmission.

\section{SIGNIFICANCE STATEMENT}

The novel small-molecule psychomotor stabilizer, IRL790, is currently in clinical trial for treatment of levodopa-induced dyskinesia and psychosis in patients with Parkinson disease. In this study, we report that systemically administered IRL790 rapidly elicits changes in protein level and phosphorylation state of proteins associated with a beneficial effect on synaptic markers and neurotransmission.

ABBREVIATIONS: AD, Alzheimer disease; Arc, activity-regulated cytoskeleton-associated protein; BDNF, brain-derived neurotrophic factor; CREB, CAMP response element-binding protein; DA, dopamine; HD, Huntington disease; LID, levodopa-induced dyskinesias; PD, Parkinson disease; TBS, Tris-buffered saline; TF, transcription factor; TH, tyrosine hydroxylase; TrkB, tropomyosin receptor kinase B. 
The overall goal of this study was to investigate the preclinical pharmacology of IRL790 in mice and its effects on biochemical measures of synaptic transmission and plasticity. Changes in receptor protein level and/or phosphorylation state, signal transduction proteins, and transcription factors (TFs) implicated in synaptic plasticity might suggest mechanism of action pathways for IRL790. We performed biochemical measurements of relevant receptors, downstream signaling targets, and TFs that were selected based on 1) the properties of the IRL790 compound, 2) the screening procedure that was applied in the development of the IRL790 compound, and 3) neuromolecular changes that have been associated with LID (Jenner, 2008). To characterize the effects of this compound, we thus investigated the phosphorylation state and protein levels of intracellular proteins, receptors, and TFs involved in the dopaminergic, glutamatergic, BDNF, and MEK/MAPK pathways that are relevant for synaptic plasticity and transmission (Qi et al., 2009).

\section{Material and Methods}

Mice. Fourteen-week-old male C57BL/J mice were purchased from Charles River and used for the described experiments. The mice were housed five per cage under a normal light/dark cycle (7 AM to $7 \mathrm{PM}$ ) in a clean facility (temperature $20-23^{\circ} \mathrm{C}$ and humidity $40 \%-60 \%$ ) and with free access to water and standard rodent chow. Mice were acclimatized for 1 week before experiments were performed. All experiments were approved by the local ethical committee in Stockholm and in agreement with the European Community Council Directive of November 24, 1986 (86/609/EEC), on the ethical use of animals.

Drug Administration and Sample Collection. IRL790 ([2-(3fluoro-5-methanesulfonylphenoxy)ethyl](propyl)amine) was synthesized at Integrative Research Laboratories, Sweden (Waters et al., 2020). The dose range was selected based on prior experience in rats and mice (in communication with Integrative Research Laboratories) to be pharmacologically relevant, i.e., to capture typical behavioral and neurochemical effects, and to be in a well tolerated range. A total of $3-10 \mathrm{mg}$ is well tolerated in mice and was considered pharmacologically relevant based on allometric scaling based on rat dosages. Two separate experiments were performed. In the first experiment, mice ( $n=6$ per treatment group) were injected intraperitoneally with IRL790 (3 or $10 \mathrm{mg} / \mathrm{kg}$ ) or sterile saline. One group of mice was injected with the $\mathrm{D}_{2}$ antagonist haloperidol $2 \mathrm{mg} / \mathrm{kg}$ (Janssen, Denmark) as a control for correctly performed injections $(n=4)$. Haloperidol results were thus compared with previously published results when available. Mice were euthanized 30 minutes postinjection by cervical dislocation followed by decapitation, and the brain was removed and snap-frozen in isopentane cooled with dry ice. Dissection of striatum and prefrontal cortex was performed for each animal, and samples were stored at $-80^{\circ} \mathrm{C}$ and subsequently prepared for protein analysis. In the second experiment, mice were injected intraperitoneally with IRL790 ( $3 \mathrm{mg} / \mathrm{kg})$, haloperidol $(2 \mathrm{mg} / \mathrm{kg})$, or saline $(n=6$ per treatment group) and euthanized 15 or 30 minutes after drug administration. Striatum and prefrontal cortex tissues were collected for each animal as described in the first experiment.

Open-Field Test. Mice in the first experiment were tested in an open-field test to assay locomotor activity. Mice were treated according to the different treatment regimens, and 20 minutes postinjection, they were placed in a $55-\mathrm{cm} \times 55$-cm squared box for 10 minutes. Total distance traveled, average speed, and time spent in the center zone were endpoints measured using an automated video tracking system (Ethovision XT 8; Noldus, Wageningen, Netherlands).

Western Blot Analysis. Protein was extracted from fresh-frozen striatum, and prefrontal cortical samples were collected and stored in $-80^{\circ} \mathrm{C}$. Tissue samples were lysed in $1 \%$ SDS and homogenized using sonication (Epishear Probe sonicator; Active motif). Samples were boiled for 10 minutes and then centrifuged for 10 minutes at 10,000 rpm. Protein concentration was determined using the BCA assay (Pierce BCA protein assay kit; Thermoscientific) according to the manufacturer's protocol. Loading sample buffer was added to each sample following quantification. For analysis, $30 \mu \mathrm{g}$ protein of each sample was separated on acrylamide gels by using the Bio-Rad system. Different running gel concentrations were used depending on the molecular size of the investigated protein, ranging from $7.5 \%$ for high molecular weight proteins $(200 \mathrm{kDa})$ to $15 \%$ for low molecular weight proteins $(10-20 \mathrm{kDa})$. Gels were first run at $120 \mathrm{~V}$ for 10 minutes and then $160 \mathrm{~V}$ for 50-80 minutes. Proteins were then transferred to an Immobilion-FL polyvinylidene difluoride (PVDF) membrane (Millipore) overnight at $50 \mathrm{~mA}$. The membrane was blocked in $5 \%$ (w/v) dry milk in TBS/Tween 20 for 1 hour at room temperature, followed by primary antibody incubation. Membranes were incubated overnight at $+4^{\circ} \mathrm{C}$ with specific primary antibodies diluted in 5\% dry milk dissolved in TBS/Tween 20. After washing with TBS/Tween 20, membranes were incubated 1 hour at room temperature with specific anti-mouse or anti-goat horseradish peroxidase secondary antibodies, diluted 1:6000 in 5\% dry milk in TBS/Tween 20. Immunoblotting was carried out with phosphorylation state-specific antibodies and with antibodies that are not phosphorylation statespecific. Immunoreactive proteins were visualized by using the chemiluminescence ECL detection system (GE Healthcare) and autoradiography or fluorescent detection by using secondary antibodies covalently labeled with fluorescent dyes in the near-infrared spectrum. The following protein targets were analyzed using the ECL system: anti-phospho-Ser ${ }^{845}$ and anti-phospho-Ser ${ }^{831}$ GluR1, antiphospho-Ser ${ }^{896}$ and anti-phospho-Ser ${ }^{897}$ NR1, anti-phospho-Ser ${ }^{473}$ AKT and anti-phospho-Thr ${ }^{308}$ AKT, anti-phospho-Tyr ${ }^{816}$ TrkB, antiArc, anti-proBDNF, anti-BDNF, anti-GluR1, anti-NR1, anti-AKT, and anti-TrkB. Antibody dilutions used 1:500-1:2000. Densitometry was performed using the ImageJ software (National Institutes of Health). Combined detection of total protein and phospho-protein using quantitative infrared Western blot analysis was applied on the following targets: phospho-Ser ${ }^{19}$, phospho-Ser ${ }^{31}$ and phospho-Ser ${ }^{40}$ tyrosine hydroxylase (TH), total TH, phospho-Ser ${ }^{1303}$ NR2B, total NR2B, phospho-Ser ${ }^{133}$ CREB, total CREB, phospho-Ser ${ }^{217 / 221}$ MEK, total MEK, phospho-Ser ${ }^{9}$ GSK3 $\beta$, total GSK3 $\beta$, phospho-Thr ${ }^{286 / 287}$ CAMKII, total CAMKII, phospho-Thr ${ }^{183 / 185}$ (p42) and phospho$\mathrm{Thr}^{202 / 204}$ (p44) MAPK, total MAPK, phospho-Thr ${ }^{34}$, phospho-Thr ${ }^{75}$ DARPP-32, and total DARPP-32. The membranes were coincubated with specific primary rabbit anti-phospho antibodies and mouse primary antibodies specific for the total protein. Membranes were first incubated with the primary antibodies diluted in 5\% dry milk in TBS/Tween 20. Proteins were detected with a mixture of infrared dye $800 \mathrm{CW}$ goa-anti-rabbit IgG $(1: 20,000)$ and infrared dye $680 \mathrm{CW}$ goatanti--mouse IgG $(1: 25,000)$ labeled secondary antibodies diluted in $0.01 \%$ SDS/5\% dry milk in TBS/Tween 20 for 1 hour at room temperature in dark conditions. The LiCor Odyssey CLx Infrared Imaging system was used for signal detection. Protein quantification was performed by using the Image Studio version 3.1 software. Levels of phospho-protein, normalized to total protein levels in the same well, were quantified by analysis of fluorescence intensities. Calnexin or Actin (used for anti-phospho-Ser ${ }^{896}$, anti-phospho-Thr ${ }^{308}$, anti-phospho-Ser ${ }^{43}$, total NR1, total Akt, and anti-phospho-Ser ${ }^{897}$ NR1) were used as loading control (housekeeping protein) and run on the same gel as the target protein being analyzed. The experimental conditions did not have any effect on the protein levels of the housekeeping proteins (data not shown). Normalization was performed for all samples. For the multiplex fluorescent detection, the phosphospecific signal was normalized against the total level of the target protein, using the target protein as its own internal loading control (phosphorylated protein signal/total protein signal)/percent control group. Relative total protein level was calculated as a ratio between (total protein signal/house-keeping protein signal)/percent control group. For protein targets that were analyzed using the ECL system, 
relative levels were calculated as a ratio between [(phosphorylated protein/house-keeping protein)/(total protein/house-keeping protein)]/ percent control group. For Western blotting, we analyzed $n=6$ per treatment group except for the haloperidol treated group in experiment $1(n=4)$.

Statistical Analysis. Data analysis was performed by comparing the mice treated with $3 \mathrm{mg} / \mathrm{kg}$ IRL790, $10 \mathrm{mg} / \mathrm{kg}$ IRL790, and haloperidol, respectively, with the saline-treated control group using one-way analysis of variance (ANOVA) and Dunnett's test specifically testing differences between groups treated with IRL790 versus the control group (GraphPad Prism5). We used the Dunnett method specifically for testing our data with control group design, as it is a powerful statistical method that can discover relatively small but significant differences among groups. The Dunnett's test has greater statistical power to detect a difference than the omnibus ANOVA test and thus has the power to identify statistical differences even when the ANOVA fails to do so. The Dunnett's test does not require a significant omnibus F-test before proceeding (Cardinal and Aitken, 2006). The figure legends describe the statistical analysis based on the overall ANOVA and the post hoc Dunnett's test. Data distribution was assumed to be normal, but this was not formally tested. Data are reported as mean \pm S.E.M. or box and whisker plots showing the median, with the box extending from the 25 th to the 75 th percentile and the whiskers showing the smallest value up to the largest. Bonferroni correction was not implemented in the statistical analysis of the different protein targets. We have marked the graphs as significant when the Dunnett's test has shown $P<0.05$ between control and treatment groups. $P$ values less than 0.05 were considered significant.

\section{Results}

Dose Finding. We first studied the effects of high and low dose of IRL790 on motor effects in mice because the preclinical characterization of IRL790 has been exclusively done in rat (Waters et al., 2017, 2020). Mice were treated with 3 and $10 \mathrm{mg} / \mathrm{kg}$, respectively, and tested for locomotor activity 20 minutes postadministration. Time spent in the center zone of an open field is a measure of normal exploratory behavior, whereas less time in the center zone indicates an anxiety-like behavior (Crawley, 1985). The different treatment regimens did not have an effect on the exploratory behavior (Fig. 1A). However, we observed that administration of IRL790 at the higher dose $(10 \mathrm{mg} / \mathrm{kg})$ caused hypolocomotion with significantly reduced total distance traveled (Fig. 1B) and reduced average speed (Fig. 1C) compared with the control group, whereas mice treated with lower dose $(3 \mathrm{mg} / \mathrm{kg})$ showed no difference compared with the controls. Total distance traveled and average speed was on average reduced by half but with a smaller reduction compared with haloperidol-treated mice.

IRL790 Upregulates proBDNF Protein Levels in Prefrontal Cortex. We next assessed the protein levels and the phosphorylation state of neurotrophic factors and TFs by using Western blotting for Arc, proBDNF, BDNF, TrkB, and phosphorylation at Tyr816 of TrkB and at Ser133 of the protein CREB. Our analysis showed that both proBDNF and BDNF were expressed and quantifiable in striatum and prefrontal cortex (Yang et al., 2009). Interestingly, we observed increased protein levels of proBDNF in prefrontal cortex at 30 minutes postadministration in the mice injected with $3 \mathrm{mg} / \mathrm{kg}$ of IRL790 (Fig. 2A). There was no difference in proBDNF levels in striatum (Fig. 2B) or mature BDNF protein levels in the prefrontal cortex (Fig. 2C) or striatum (Fig. 2D) between the treatment groups (Supplemental Fig. 1).
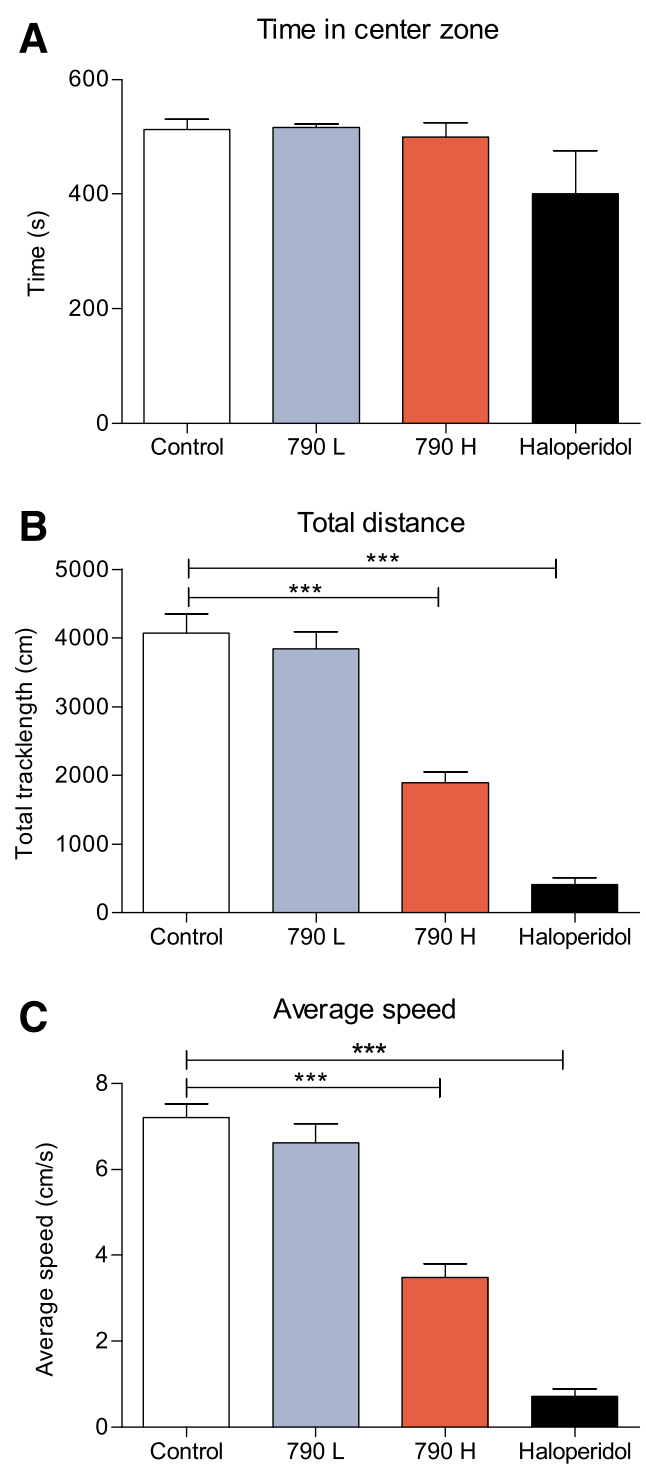

Fig. 1. High dose of IRL790 (10 mg/kg) exerts an effect on locomotor activity. Mice were treated with IRL790 (L (low dose) $=3 \mathrm{mg} / \mathrm{kg}$ and $\mathrm{H}$ (high dose $)=10 \mathrm{mg} / \mathrm{kg}$ ) and the $\mathrm{D}_{2}$ antagonist haloperidol $(2 \mathrm{mg} / \mathrm{kg})$ and tested for locomotor activity 20 minutes postadministration. (A) The IRL790 doses tested did not show any effect on anxiety-related behavior compared with the control group. IRL790 H $(10 \mathrm{mg} / \mathrm{kg})$ promoted a sedative effect similar to haloperidol with significantly reduced total distance traveled $\left(\mathrm{F}_{(3,20)}=68.00, P<0.0001\right)(\mathrm{B})$ and reduced average speed $\left(\mathrm{F}_{(3,20)}=83.85, P<0.0001\right)(\mathrm{C})$. Bars are presented as mean \pm S.E.M. $n=6$ per group; $\mathrm{n}=4$ treated with haloperidol. One-way ANOVA followed by Dunnett's multiple comparison test was used. $* * * P$ value $<0.001$

Furthermore, there were no significant differences in Arc and TrkB protein levels or the phosphorylation state at Tyr816 of TrkB and Ser133 of CREB at 30 minutes postadministration in the prefrontal cortex or striatum, respectively (Supplemental Fig. 2).

IRL790 Upregulates TH Activity and Presynaptic Regulation of Dopamine Synthesis. It has been suggested that IRL790 increases DA turnover in basal ganglia and cortical regions and extracellular DA, NE, and Ach levels as measured by in vivo microdialysis (Waters et al., 2020). Tyrosine hydroxylase (TH) is the rate-limiting enzyme of catecholamine synthesis and is phosphorylated at Ser residues Ser19, Ser31, and Ser40 through the actions of CAMKII, 
A
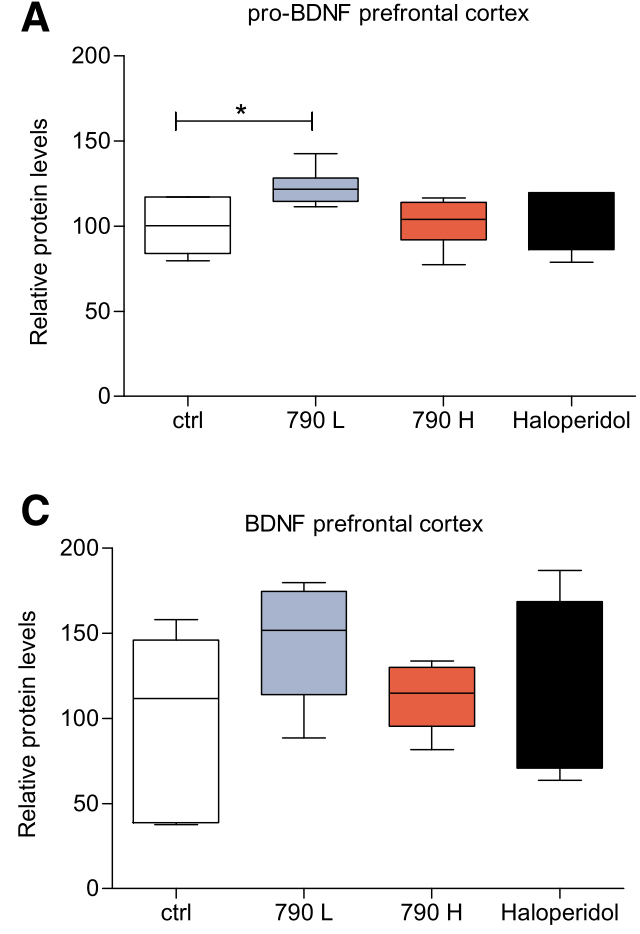

B

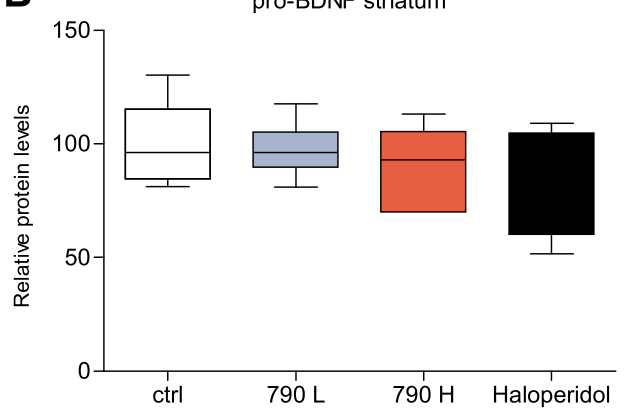

D

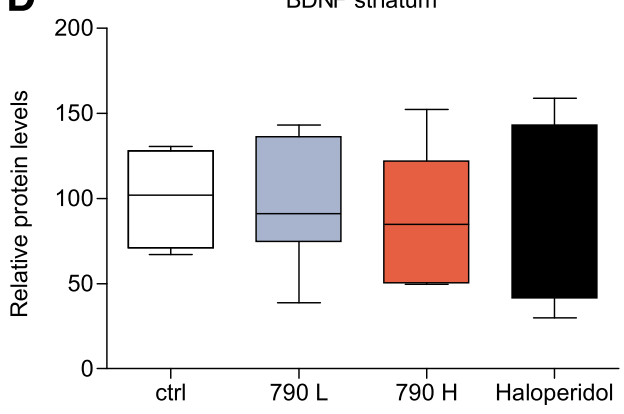

Fig. 2. Acute administration of IRL790 upregulated proBDNF protein levels in prefrontal cortex. (A) The levels of proBDNF in prefrontal cortex increased in the mice injected with IRL790 $(3 \mathrm{mg} / \mathrm{kg})$ at 30 minutes postadministration $\left(\mathrm{F}_{(3,18)}=2.94, P=0.061\right)$ as assessed by immunoblotting. The overall $P$ value for the ANOVA was not significant; however, Dunnett's test, specifically comparing groups on IRL790 vs. controls, indicated a significant difference between mice treated with IRL790 $(3 \mathrm{mg} / \mathrm{kg})$ and controls. There was no difference in proBDNF protein levels in striatum (B), mature BDNF in prefrontal cortex (C), or mature BDNF in striatum (D) in mice treated with 3 and $10 \mathrm{mg} / \mathrm{kg}$ IRL790 and controls ( $n=6$ per group; $n=4$ treated with haloperidol). ctrl, control. $\mathrm{L}=$ low dose, $3 \mathrm{mg} / \mathrm{kg}$ and $\mathrm{H}=$ high dose, $10 \mathrm{mg} / \mathrm{kg}$. $* P$ value $<0.05$
Extracellular signal-regulated protein kinases 1 and 2 (ERK1/ 2), and protein kinase A (PKA), respectively (Salvatore et al., 2001; Dunkley et al., 2004). We next used two-color detection and quantification of infrared fluorescent Western blotting to assess the phosphorylation levels of TH because of its critical involvement in the presynaptic synthesis of catecholamine. We assessed the striatum, which is the largest component of the basal ganglia and part of the dopaminergic pathway. There was a significant increase in phosphorylation at Ser40 in striatum in mice treated with $10 \mathrm{mg} / \mathrm{kg}$ of IRL 790 compared with the controls 30 minutes postadministration (Fig. 3A; Supplemental Fig. 3). The phosphorylation state at Ser31 in the striatum was significantly increased in mice treated with 3 or $10 \mathrm{mg} / \mathrm{kg}$ of IRL790 compared with the controls, 30 minutes postadministration (Fig. 3B). We also observed an increase in phosphorylation at Ser19 in striatum in mice treated with $10 \mathrm{mg} / \mathrm{kg}$ of IRL790 compared with the controls at 30 minutes postadministration (Fig. 3C). The total levels of TH were not different between the treatment groups (Fig. 3D). Hence, we found that IRL790 increased phosphorylation at all serine residues that directly or indirectly associate with increased TH activity.

Acute Administration of IRL790 Exerts No Effect on Postsynaptic Regulation of Glutamate Signaling. We next assessed the phosphorylation state of AMPA and NMDA receptors, as they associate with various states of synaptic plasticity e.g., long-term potentiation and long-term depression (Derkach et al., 2007; Paoletti et al., 2013). At the studied doses, there were no changes in phosphorylation at Ser831 and Ser845 on the AMPA GluR subunit, the Ser896, Ser897 on the NR1 subunit, or the Ser1303 on the NR2B subunit between IRL790-treated groups and controls at 30 minutes postadministration in the striatum (Supplemental Fig. 4) or cortical tissues (Supplemental Fig. 5), respectively. However, we observed reduced total protein levels of the NR2B subunit in the striatum of mice treated with a $3-\mathrm{mg} / \mathrm{kg}$ dose of IRL790 (Fig. 3E) but not in the prefrontal cortex (Fig. 3F; Supplemental Fig. 6).

Acute Administration of IRL790 Exerts No Effect on the Phosphorylation State of Intracellular Signaling Proteins. There is a vast number of proteins that are central for intracellular signaling, of which we next assessed the phosphorylation state of proteins involved in the MEK/MAPK pathway and GSK3 $\beta$, CaMKII, and Akt signaling, including Ser217/221-MEK, Thr183/Tyr185-p42 MAPK, Thr202/ Tyr204-p44 MAPK, Ser9-GSK3 $\beta$, Thr286-CaMKII $\beta$, Thr287CaMKII $\alpha$, Thr308-Akt, Ser473-Akt. Acute systemic administration of IRL790, however, had no overt effects on any of these phosphorylation sites at 30 minutes postadministration in striatum (Supplemental Fig. 7) or prefrontal cortex (Supplemental Fig. 8), respectively. Dopamine- and cAMPregulated neuronal phosphoprotein (DARPP32) is a target for dopamine, which has the ability to modulate the activity of protein phosphatase 1 (PP-1) and PKA, regulating physiologic and pharmacological stimuli (Svenningsson et al., 2004). We measured the phosphorylation of DARPP32 at both Thr34 and Thr75 in striatum but observed no difference in phosphorylation state between the controls and IRL790-treated mice (Supplemental Fig. 9).

Rapid Phosphorylation Changes Induced by IRL790. We next wanted to investigate if there were any effects at an earlier time point by administering the same dose of $3 \mathrm{mg} / \mathrm{kg}$ of IRL790 and by analyzing the exact same panel of proteins using Western blotting. In addition to assessing phosphorylation states already at 15 minutes postadministration, we also repeated the 30-minute time point to validate our initial findings in a separate experiment. Data are here only shown for the proteins with significant differences between treatment groups following 15 minutes of IRL790 administration (dose $3 \mathrm{mg} / \mathrm{kg}$ ). Phosphorylation at Ser31 of TH was significantly increased in striatum in mice treated with $3 \mathrm{mg} / \mathrm{kg}$ of IRL790 compared with the controls already at 15 minutes 

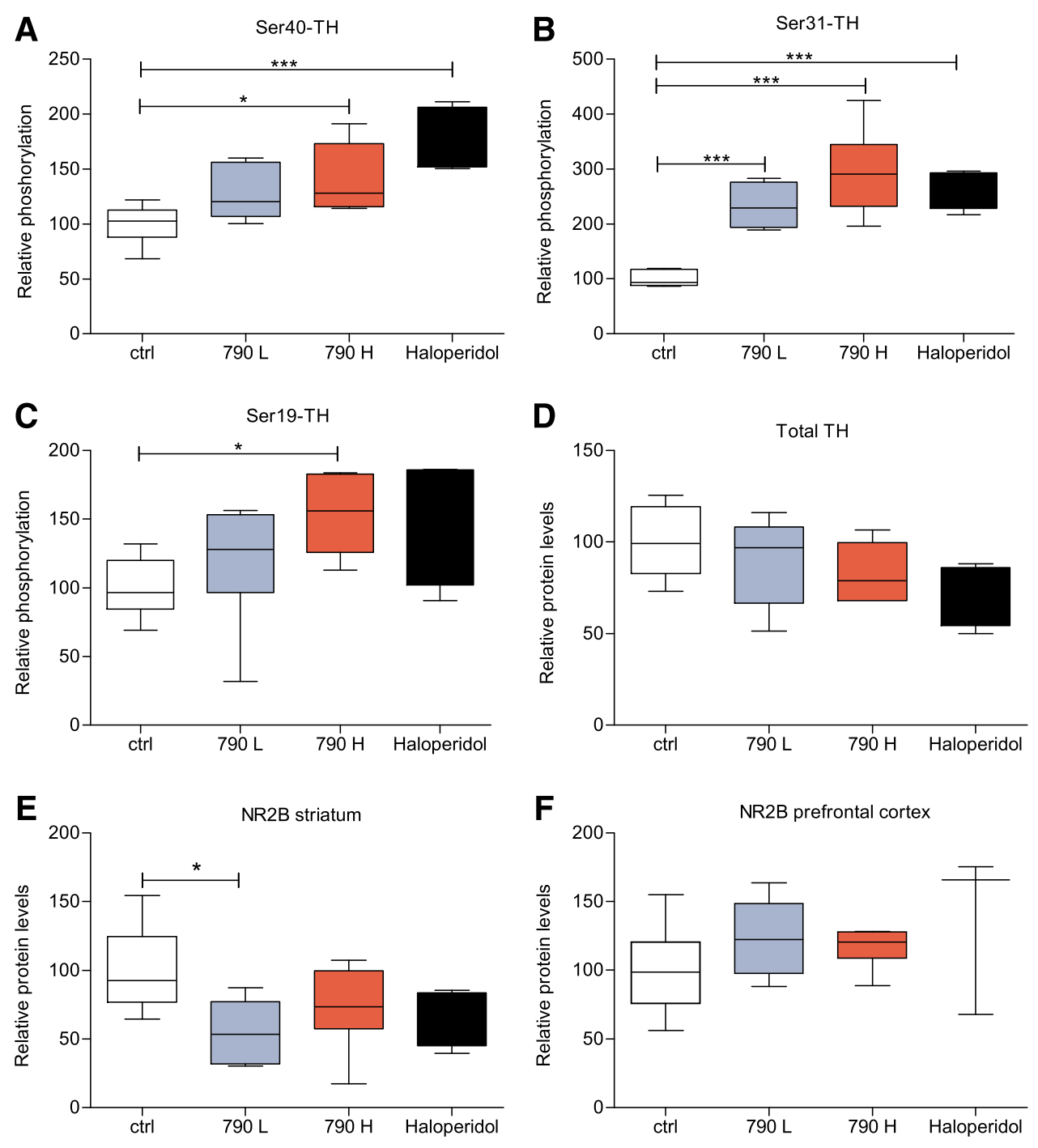

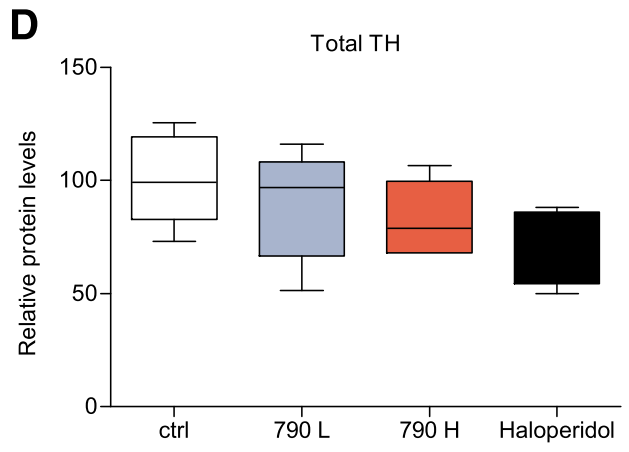

postadministration (Fig. 4A) and at 30 minutes postadministration of IRL790, which validated our result in the first experiment (Fig. 4B; Supplemental Fig. 10, A and B). There was no statistical difference in phosphorylation at Ser19 and Ser41 of TH at 15 minutes of treatment (data not shown). We observed a significant increase in phosphorylation at Ser1303 of the NR2B at 15 minutes postadministration in the prefrontal cortex compared with the controls (Fig. 4C; Supplemental Fig. 10, C and D). Notably, there was no change in phosphorylation of NR2B at Ser1303 at 30 minutes postadministration, thus confirming the observation in the first experiment (Fig. 4D). In addition, there was no increase in proBDNF or BDNF protein levels in prefrontal cortex or striatum at 15 minutes postadministration of IRL790 (3 $\mathrm{mg} / \mathrm{kg}$ ). We next combined all data from the two separate experiments (experiment 1 and 2) with 30-minute administration of IRL790 $3 \mathrm{mg} / \mathrm{kg}$ to increase the sample size. Pooled data showed that proBDNF (Fig. 4E) protein levels were significantly increased in the prefrontal cortex of IRL790treated mice but not mature BDNF levels (Fig. 4F). As an additional observation, haloperidol increased Ser845 phosphorylation on the AMPA GluR1 subunit in striatum, with the greatest increase observed at 15 minutes but still elevated at 30 minutes (data not shown), confirming previous studies,
Fig. 3. Acute administration of IRL790 increased phosphorylation at Ser40, Ser31, and Ser19 of TH in striatum. (A) Phosphorylation increased at Ser40 in mice treated with IRL790 $(10 \mathrm{mg} / \mathrm{kg})$ compared with the controls 30 minutes postadministration $\left(\mathrm{F}_{(3,18)}=7.40, P=\right.$ 0.002 ) as assessed by immunoblotting. (B) Phosphorylation was increased at Ser31 in mice treated with both 3 and $10 \mathrm{mg} / \mathrm{kg}$ of IRL790 compared with the controls $\left(\mathrm{F}_{(3,18)}=17.91, P<0.00001\right)$. $(\mathrm{C})$ Phosphorylation at Ser19 was increased in mice treated with IRL790 $(10 \mathrm{mg} / \mathrm{kg})$ compared with the controls $\left(\mathrm{F}_{(3,18)}=2.86\right.$, $P=0.066$ ). (D) The protein levels of total TH showed no difference between treatment groups. Data corresponding to total TH levels based on assessment of phosphorylation at Ser40 is shown here. (E) The total protein levels of the NR2B subunit were reduced in striatum in mice treated with IRL790 $(3 \mathrm{mg} / \mathrm{kg})$ at 30 minutes postadministration $\left(\mathrm{F}_{(3,18)}=\right.$ $2.77, P=0.071)$, but not in prefrontal cortex $(\mathrm{F})$ as assessed by immunoblotting. The overall $P$ value for the ANOVA was not significant for analysis of total NR2B levels in striatum. However, Dunnett's test, specifically comparing groups on IRL790 vs. controls, indicated a significant difference between mice treated with IRL790 $(3 \mathrm{mg} / \mathrm{kg})$ and controls $(n=6$ per group; $n=4$ treated with haloperidol; $n=$ 3 treated with haloperidol in prefrontal cortex). ctrl, control. $\mathrm{L}=$ low dose, $3 \mathrm{mg} / \mathrm{kg}$ and $\mathrm{H}$, high dose $=10 \mathrm{mg} / \mathrm{kg}$. $* P$ value $<$ $0.05, * * * P$ value $<0.001$

which have shown that haloperidol increases Ser845 but not Ser831 phosphorylation at GluR1 (Håkansson et al., 2006).

\section{Discussion}

In this study, we investigated the effects of the novel small molecule, IRL790, on synaptic markers. First, we showed a difference on locomotor activity between the high and low dose of IRL790, and we showed that mice treated with IRL790 displayed no anxiety-like behavior. Our findings further provide evidence that IRL790 exerts biochemical changes in naïve mouse brain, including increased proBDNF protein levels in prefrontal cortex and increased phosphorylation of serine residues that associate with increased $\mathrm{TH}$ activity in striatum. Furthermore, we showed that compared with controls, the total levels of NR2B were reduced in striatum of mice treated with IRL790 and that IRL790 rapidly (already at 15 minutes postdrug administration) phosphorylated the NR2B in the prefrontal cortex.

Our data suggest that rapid upregulation of proBDNF protein levels in prefrontal cortex constitutes one molecular mechanism of action of IRL790. proBDNF is the precursor of $\mathrm{BDNF}$, where the latter has emerged as an essential regulator of synaptic plasticity, neuronal survival, and differentiation, 

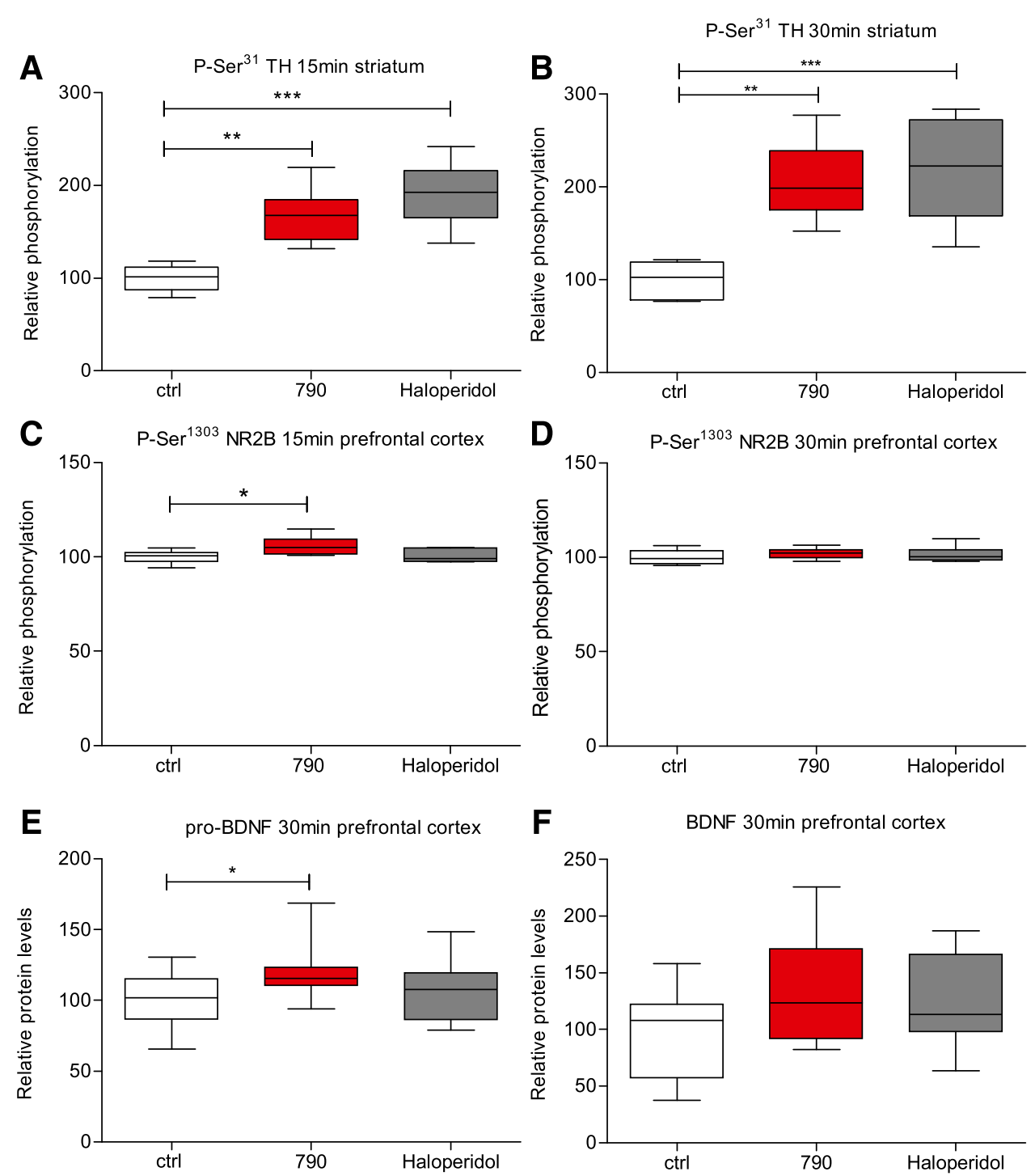

Fig. 4. Acute administration of IRL790 induces rapid phosphorylation changes. (A) Phosphorylation at Ser31 of TH in striatum increased in mice treated with IRL790 $(3 \mathrm{mg} / \mathrm{kg})$ compared with the controls already at 15 minutes postadministration as assessed by immunoblotting $\left(\mathrm{F}_{(2,15)}=16.98, P=0.0001\right)$ and $(\mathrm{B})$ 30 minutes postadministration $\left(\mathrm{F}_{(2,15)}=\right.$ $14.42, P=0.0003$ ). (C) There was a significant increase in phosphorylation at Ser1303 of the NR2B at 15 minutes postadministration in prefrontal cortex in the IRL790 $(3 \mathrm{mg} / \mathrm{kg})$ treated mice compared with the controls $\left(\mathrm{F}_{(2,15)}=3.70, P=\right.$ 0.049 ). (D) There was no change in phosphorylation of NR2B at Ser1303 at 30 minutes postadministration $(n=6$ per group). (E) Pooling of data from the first and the second experiment confirmed a significant increase in proBDNF protein levels $\left(\mathrm{F}_{(2,31)}=3.09, P=0.060\right)$, but not BDNF levels (F), in the prefrontal cortex of IRL790-treated mice compared with controls $(n=12$ per group; $n=10$ for haloperidol treated mice). The overall $P$ value for the ANOVA was not significant; however, Dunnett's test, specifically comparing groups treated with IRL790 vs. controls, indicated a significant difference between low-dose IRL790-treated mice $(3 \mathrm{mg} / \mathrm{kg})$ and controls. ctrl, control. $\mathrm{L}=$ low dose, $3 \mathrm{mg} / \mathrm{kg}$ and $\mathrm{H}=$ high dose, $10 \mathrm{mg} / \mathrm{kg}$.* $P$ value $<0.05$, ** $P$ value $<0.01$, *** $P$ value $<0.001$ especially in areas of the brain that are associated with learning, memory, and higher thinking (Cunha et al., 2010). BDNF activity is further associated with increased long-term potentiation that improves learning and memory by strengthening the communication between specific neurons (Figurov et al., 1996). Interestingly, studies have provided evidence implicating BDNF in the pathogenesis of neurodegenerative diseases, such as Huntington disease (HD), Alzheimer disease (AD), and PD (Zuccato and Cattaneo, 2009). For example, multiple studies have shown that BDNF levels are reduced in $\mathrm{HD}, \mathrm{AD}$, and $\mathrm{PD}$ postmortem brain studies (Connor et al., 1997; Holsinger et al., 2000; Peng et al., 2005a) and that BDNF depletion contributes to neurodegeneration in the brain (Zuccato and Cattaneo, 2009). Wnt-BDNF ${ }^{\mathrm{KO}}$ mice lacking BDNF in the midbrain were shown to have a reduction of dopaminergic neurons in substantia nigra ((Baquet et al., 2005), whereas mice treated with antisense oligonucleotides targeting BDNF displayed impaired behavior (Porritt et al., 2005). Accordingly, preclinical studies have shown beneficial effects of increasing BDNF levels, which has made BDNF an attractive therapeutic target for neurodegenerative diseases (Zuccato and Cattaneo, 2009). However, studies have shown that L-dopa treatment induces sprouting of serotonin axons and increased BDNF protein levels in the striatum and motor/ premotor cortices in the 6-OHDA rat model of PD (Rylander et al., 2010). Moreover, a recent study by Tronci et al. showed that BDNF overexpression induced sprouting of serotonin neuron terminals and exacerbation of LID, suggesting a pathophysiological role of BDNF in 6-OHDA lesioned rats (Tronci et al., 2017). These results are of importance considering that IRL790 is developed to treat LID and psychosis, and we here show that IRL790 upregulates proBDNF protein levels in prefrontal cortex. However, results have been reported showing that IRL790 alleviated LID without affecting levodopainduced therapeutic effects (Waters et al., 2020).

Phosphorylation of TH at Ser40 increases TH activity, whereas phosphorylation at Ser31 also increases TH activity but to a lesser extent (Dunkley et al., 2004). The phosphorylation of TH at Ser19 exerts no direct effect on TH activity. Instead, it increases the rate of Ser40 phosphorylation, leading to increased $\mathrm{TH}$ activity. Of note is that treatments that stimulate Ser31 phosphorylation alone may lead to increased dopamine biosynthesis (Mitchell et al., 1990). Studies have found that TH activity was reduced in the brains 
of $\mathrm{AD}$ patients compared with healthy individuals (Sawada et al., 1987) and that alterations in the enzymatic activity of TH may be involved in disorders such as PD and schizophrenia because of its rate-limiting role in the biosynthesis of catecholamines, catalyzing the hydroxylation of L-tyrosine to DOPA (Nagatsu and Sawada, 2007). Interestingly, early studies found reduction of $\mathrm{TH}$ expression and decreased $\mathrm{TH}$ activity in PD brains in the nigro-striatum as well as in the adrenal medulla, which is considered to contribute to catecholeamine deficiency (Nagatsu and Sawada, 2007). The molecular activity, i.e., homo-specific activity, however, was increased in PD brains, suggesting a compensatory increase in TH activity (Mogi et al., 1988). It is, however, not clear when the observed enzyme changes occur in relation to the neuronal degeneration of the catecholamine neurons in PD. Interestingly, $\alpha$-synuclein has been shown to interact with $\mathrm{TH}$ and to repress TH activity, not only by inhibiting phosphorylation at Ser40 of $\mathrm{TH}$ but also by stimulating protein phosphatase $2 \mathrm{~A}$ activity, resulting in decreased dopamine synthesis (Perez et al., 2002; Peng et al., 2005b). In this context it is also worth mentioning 14-3-3 proteins that have been associated with PD based on their presence in Lewy bodies and binding to PD-associated proteins, including TH (Foote and Zhou, 2012). TH is activated by phosphorylation-dependent (Ser19 and Ser40) binding to 14-3-3 proteins (Kleppe et al., 2001). In contrast to $\alpha$ synuclein, the 14-3-3 proteins are known to exert a neuroprotective effect by promoting cell survival by inhibiting apoptotic processes via multiple mechanisms (Foote and Zhou, 2012). For example, the beneficial effects of 14-3-3 proteins have been shown in cellular toxin-induced models of PD. Intricate interactions between these three proteins may thus be important in the regulation of dopamine biosynthesis in PD. Therapeutic agents that increase the intrastriatal TH activity and maintain relevant DA concentrations are hypothetically expected to alleviate PD symptoms. Thus, although our observed effects of IRL790 on TH phosphorylation states suggest potential for increased $\mathrm{TH}$ activity, we need to further investigate if increasing the phosphorylation at Ser19 and Ser40 of TH induced by IRL790 might help increase the $\mathrm{TH}$ activity and dopamine synthesis in PD.

NMDARs are essential for excitatory neurotransmission and synaptic plasticity in the nervous system. Our results showed that IRL790 elicited rapid changes with increased phosphorylation at Ser1303 of NR2B in prefrontal cortex. In addition, IRL790 induced downregulation of total protein levels of the NR2B subunit in the striatum of mice treated with IRL790. PKA, PKC, and especially CAMKII activity induces the phosphorylation at Ser1303 of NR2B, which is associated with increased $\mathrm{Ca}^{2+}$ and is critical for synaptic plasticity, learning, and memory (Salter et al., 2009). In contrast, alterations in NMDAR function and in the balance between downstream kinases and phosphatases can occur in pathologic conditions and lead to neuronal excitotoxicity. Studies indicate that changes in glutamatergic transmission are implicated in disorders such as $\mathrm{HD}, \mathrm{PD}$, and schizophrenia (Loftis and Janowsky, 2003) and that compounds that modulate the transmission at NMDA receptors have therapeutic potential in PD (Johnson et al., 2009) and HD (Frank and Jankovic, 2010). Moreover, the dopaminergic stabilizer pridopidine is suggested to exert its positive effects through modulation of both dopamine transmission and activation of cortical glutamate transmission through NMDA receptors (Ponten et al., 2010).

Previous work studying the effects of IRL790 in rats showed that the IRL790 profile is distinct from strong, conventional DA D2 blockers such as haloperidol (Waters et al., 2017). In our experimental setting, haloperidol increased phosphorylation at Ser845 on the AMPA GluR1 subunit in striatum, similarly to previous studies using lower doses, which have shown that haloperidol increases Ser845 but not Ser831 phosphorylation (Håkansson et al., 2006). Furthermore, there are studies showing effects of haloperidol on the MEK/MAPK pathways, although these show dose effects of the drug. However, our results are in line with previous studies showing that higher doses of haloperidol ( 2 and $4 \mathrm{mg} / \mathrm{kg}$ ) exerted no effects on phosphorylation state of the MEK/MAPK proteins (p-Ser217/221-MEK1/2, p-Thr202/Tyr204-ERK1/2) compared with 0.5 and $1 \mathrm{mg} / \mathrm{kg}$, which lead to biphasic phosphorylation responses (Kim et al., 2008). Nevertheless, discrepancies between our results and other studies likely reflect kinetics and dose-response effects, which thus need to be accounted for when interpreting the results reported in this study. Furthermore, we focused our efforts on measuring the biochemical effects of IRL790 in naïve mouse brain, whereas previous studies used the rat as study model. Although some biochemical parameters such as absorption may be reasonably extrapolated between different species (e.g., mice, rats) and from animals to humans, other parameters might be less predictable (e.g., protein binding and drug metabolism) (Lin, 1995). In addition, it is important to note that the effects of IRL790 might be different in a pathologic state given its characteristic psychomotor stabilizing effects (Waters et al., 2017). Hence, these biochemical aspects should be taken into consideration, as the changes we found in mice might differ in other species, including humans, and should thus be subject to further investigation.

IRL790 is developed for treatment of complications associated with therapy in PD, primarily LIDs. In fact, there is preliminary data that IRL790 counteracts LIDs in PD patients (Svenningsson et al., 2018), and IRL790 shows clear antidyskinetic effects in rats (Waters et al., 2020). Studies have reported a higher mortality rate toward dopaminergic lesioning by 6 -hydroxydopamine in mice than rats (Lundblad et al., 2004). It is therefore more cumbersome to reproducibly study LIDs in mice than rats, so we did not attempt to perform LID studies here.

In summary, this study shows the effects of IRL790, a novel small molecule with psychomotor stabilizing properties in vivo, in naïve mouse brain by assessing its effects on proteins with implications in synaptic plasticity and transmission. Our results demonstrated rapid stimulatory effects of IRL790 on proBDNF protein levels, TH activity, and NR2B phosphorylation, hence providing valuable information to the ongoing clinical studies investigating the effects of IRL790.

\section{Acknowledgments}

The authors wish to thank Maria Gullme for providing the IRL790 compound, Dr. Susanna Waters for critical discussion, and Dr. Nicoletta Schintu for technical assistance.

\section{Authorship Contributions}

Participated in research design: Becanovic, Svenningsson. Conducted experiments: Becanovic, Vittoria de Donno, Sousa. 
Contributed new reagents or analytic tools: Tedroff.

Performed data analysis: Becanovic, Vittoria de Donno.

Wrote or contributed to the writing of the manuscript: Becanovic.

\section{References}

Baquet Z C, Bickford P C, and Jones K R (2005) Brain-Derived Neurotrophic Factor Is Required for the Establishment of the Proper Number of Dopaminergic Neurons in the Substantia Nigra Pars Compacta. The Journal of neuroscience 25 (26): 6251-6259, doi: 10.1523/JNEUROSCI.4601-04.2005 15987955.

Cardinal RN and Aitken MRF (2006) Further analysis: after the ANOVA has been run, ANOVA for the Behavioral Sciences Researcher p 89, Lawrence Erlbaum Associates Inc., Mahwah, NJ.

Connor B, Young D, Yan Q, Faull RL, Synek B, and Dragunow M (1997) Brainderived neurotrophic factor is reduced in Alzheimer's disease. Brain Res Mol Brain Res 49:71-81.

Crawley JN (1985) Exploratory behavior models of anxiety in mice. Neurosci Biobehav Rev 9:37-44.

Cunha C, Brambilla R, and Thomas KL (2010) A simple role for BDNF in learning and memory? Front Mol Neurosci 3:1.

Derkach VA, Oh MC, Guire ES, and Soderling TR (2007) Regulatory mechanisms of AMPA receptors in synaptic plasticity. Nat Rev Neurosci 8:101-113.

Dunkley PR, Bobrovskaya L, Graham ME, von Nagy-Felsobuki EI, and Dickson PW (2004) Tyrosine hydroxylase phosphorylation: regulation and consequences. J Neurochem 91:1025-1043.

Figurov A, Pozzo-Miller LD, Olafsson P, Wang T, and Lu B (1996) Regulation of synaptic responses to high-frequency stimulation and LTP by neurotrophins in the hippocampus. Nature 381:706-709.

Foote M and Zhou Y (2012) 14-3-3 proteins in neurological disorders. Int J Biochem Mol Biol 3:152-164.

Frank S and Jankovic J (2010) Advances in the pharmacological management of Huntington's disease. Drugs 70:561-571.

Håkansson K, Galdi S, Hendrick J, Snyder G, Greengard P, and Fisone G (2006) Regulation of phosphorylation of the GluR1 AMPA receptor by dopamine D2 receptors. J Neurochem 96:482-488.

Holsinger RM, Schnarr J, Henry P, Castelo VT, and Fahnestock M (2000) Quantitation of BDNF mRNA in human parietal cortex by competitive reverse transcription-polymerase chain reaction: decreased levels in Alzheimer's disease. Brain Res Mol Brain Res 76:347-354.

Jenner P (2008) Molecular mechanisms of L-DOPA-induced dyskinesia. Nat Rev Neurosci 9:665-677.

Johnson KA, Conn PJ, and Niswender CM (2009) Glutamate receptors as therapeutic targets for Parkinson's disease. CNS Neurol Disord Drug Targets 8:475-491.

Kapur S and Seeman P (2000) Antipsychotic agents differ in how fast they come off the dopamine D2 receptors. Implications for atypical antipsychotic action. J Psychiatry Neurosci 25:161-166.

Kim SH, Seo MS, Jeon WJ, Yu HS, Park HG, Jung GA, Lee HY, Kang UG, and Kim YS (2008) Haloperidol regulates the phosphorylation level of the MEK-ERKp90RSK signal pathway via protein phosphatase $2 \mathrm{~A}$ in the rat frontal cortex. Int $J$ Neuropsychopharmacol 11:509-517.

Kleppe R, Toska K, and Haavik J (2001) Interaction of phosphorylated tyrosine hydroxylase with 14-3-3 proteins: evidence for a phosphoserine 40-dependent association. J Neurochem 77:1097-1107.

Lin JH (1995) Species similarities and differences in pharmacokinetics. Drug Metab Dispos 23:1008-1021.

Loftis JM and Janowsky A (2003) The N-methyl-D-aspartate receptor subunit NR2B localization, functional properties, regulation, and clinical implications. Pharmacol Ther 97:55-85.

Lundblad M, Picconi B, Lindgren H, and Cenci MA (2004) A model of L-DOPAinduced dyskinesia in 6-hydroxydopamine lesioned mice: relation to motor and cellular parameters of nigrostriatal function. Neurobiol Dis 16:110-123.

Mitchell JP, Hardie DG, and Vulliet PR (1990) Site-specific phosphorylation of tyrosine hydroxylase after $\mathrm{KCl}$ depolarization and nerve growth factor treatment of PC12 cells. J Biol Chem 265:22358-22364.

Mogi M, Harada M, Kiuchi K, Kojima K, Kondo T, Narabayashi H, Rausch D, Riederer P, Jellinger K, and Nagatsu T (1988) Homospecific activity (activity per enzyme protein) of tyrosine hydroxylase increases in parkinsonian brain. J Neural Transm (Vienna) 72:77-82.

Nagatsu T and Sawada M (2007) Biochemistry of postmortem brains in Parkinson's disease: historical overview and future prospects. J Neural Transm Suppl 113-120.
Paoletti P, Bellone C, and Zhou Q (2013) NMDA receptor subunit diversity: impact on receptor properties, synaptic plasticity and disease. Nat Rev Neurosci 14:383-400.

Peng S, Wuu J, Mufson EJ, and Fahnestock M (2005a) Precursor form of brain-derived neurotrophic factor and mature brain-derived neurotrophic factor are decreased in the pre-clinical stages of Alzheimer's disease. J Neurochem 93:1412-1421.

Peng X, Tehranian R, Dietrich P, Stefanis L, and Perez RG (2005b) Alpha-synuclein activation of protein phosphatase $2 \mathrm{~A}$ reduces tyrosine hydroxylase phosphorylation in dopaminergic cells [published correction appears in J Cell Sci (2005) 118:4073]. J Cell Sci 118:3523-3530.

Perez RG, Waymire JC, Lin E, Liu JJ, Guo F, and Zigmond MJ (2002) A role for alpha-synuclein in the regulation of dopamine biosynthesis. $J$ Neurosci 22: 3090-3099.

Pettersson F, Pontén H, Waters N, Waters S, and Sonesson C (2010) Synthesis and evaluation of a set of 4-phenylpiperidines and 4-phenylpiperazines as D2 receptor ligands and the discovery of the dopaminergic stabilizer 4-[3-(methylsulfonyl) phenyl]-1-propylpiperidine (huntexil, pridopidine, ACR16). J Med Chem 53: 2510-2520.

Ponten H, Kullingsjö J, Lagerkvist S, Martin P, Pettersson F, Sonesson C, Waters S, and Waters N (2010) In vivo pharmacology of the dopaminergic stabilizer pridopidine. Eur J Pharmacol 644:88-95.

Porritt MJ, Batchelor PE, and Howells DW (2005) Inhibiting BDNF expression by antisense oligonucleotide infusion causes loss of nigral dopaminergic neurons. Exp Neurol 192:226-234

Qi H, Mailliet F, Spedding M, Rocher C, Zhang X, Delagrange P, McEwen B, Jay TM, and Svenningsson P (2009) Antidepressants reverse the attenuation of the neurotrophic MEK/MAPK cascade in frontal cortex by elevated platform stress; reversal of effects on LTP is associated with GluA1 phosphorylation. Neuropharmacology 56: 37-46.

Rylander D, Parent M, O'Sullivan SS, Dovero S, Lees AJ, Bezard E, Descarries L, and Cenci MA (2010) Maladaptive plasticity of serotonin axon terminals in levodopa-induced dyskinesia. Ann Neurol 68:619-628.

Salter MW, Dong Y, Kalia LV, Liu XJ, and Pitcher G (2009) Regulation of NMDA receptors by kinases and phosphatases, in Biology of the NMDA Receptor (Van Dongen AM, CRC Press, Boca Raton, FL.

Salvatore MF, Waymire JC, and Haycock JW (2001) Depolarization-stimulated catecholamine biosynthesis: involvement of protein kinases and tyrosine hydroxylase phosphorylation sites in situ. $J$ Neurochem 79:349-360.

Sawada M, Hirata Y, Arai H, Iizuka R, and Nagatsu T (1987) Tyrosine hydroxylase, tryptophan hydroxylase, biopterin, and neopterin in the brains of normal controls and patients with senile dementia of Alzheimer type. J Neurochem 48:760-764.

Svenningsson P, Johansson A, Nyholm D, Tsitsi P, Hansson F, Sonesson C, and Tedroff J (2018) Safety and tolerability of IRL790 in Parkinson's disease with levodopa-induced dyskinesia-a phase $1 \mathrm{~b}$ trial. NPJ Parkinsons Dis 4:35.

Svenningsson P, Nishi A, Fisone G, Girault JA, Nairn AC, and Greengard P (2004) DARPP-32: an integrator of neurotransmission. Annu Rev Pharmacol Toxicol 44: 269-296.

Tronci E, Napolitano F, Muñoz A, Fidalgo C, Rossi F, Björklund A, Usiello A, and Carta M (2017) BDNF over-expression induces striatal serotonin fiber sprouting and increases the susceptibility to l-DOPA-induced dyskinesia in 6 OHDA-lesioned rats. Exp Neurol 297:73-81.

Waters S, Sonesson C, Svensson P, Tedroff J, Carta M, Ljung E, Gunnergren J, Edling M, Svanberg B, Fagerberg A, et al. (2020) Preclinical pharmacology of [2-(3fluoro-5-methanesulfonylphenoxy)ethyl](propyl)amine (IRL790), a novel dopamine transmission modulator for the treatment of motor and psychiatric complications in Parkinson's disease. J Pharmacol Exp Ther 374:113-125.

Waters S, Svensson P, Kullingsjö J, Pontén H, Andreasson T, Sunesson Y, Ljung E Sonesson C, and Waters N (2017) In vivo systems response profiling and multivariate classification of CNS active compounds: a structured tool for CNS drug discovery. ACS Chem Neurosci 8:785-797.

Yang J, Siao CJ, Nagappan G, Marinic T, Jing D, McGrath K, Chen ZY, Mark W, Tessarollo L, Lee FS, et al. (2009) Neuronal release of proBDNF. Nat Neurosci 12: 113-115.

Zuccato C and Cattaneo E (2009) Brain-derived neurotrophic factor in neurodegenerative diseases. Nat Rev Neurol 5:311-322.

Address correspondence to: Dr. Kristina Becanovic, Centre for Molecular Medicine (CMM) L8:01, Department of Clinical Neuroscience, Karolinska Institutet, Stockholm 17176, Sweden. E-mail: Kristina.Becanovic@ki.se 